\title{
La Pediatría en Atención Primaria en Madrid
}

\author{
C. Sánchez Pina ${ }^{a}$, P. Hernando Helguero ${ }^{b}$, M. Lorente Miñarroc, \\ MJ. Hernández Delgadod, A. Carrasco Sanze, MJ. Geijo Rincónf, J. Rodríguez Delgadog, \\ B. Rodríguez-Moldes Vázquez \\ ${ }^{a} C S$ de Griñón, Madrid. España. \\ bCS Ramón y Cajal. Alcorcón, Madrid. España. \\ ¿CS Alpes. Madrid. España. \\ ${ }^{-}$CS Ciudad San Pablo. Coslada, Madrid. España. \\ eCS Potosí. Madrid. España. \\ fCS Margaritas. Getafe, Madrid. España. \\ sCS de Alpedrete, Madrid. España. \\ ${ }^{h} C S$ de Torres de la Alameda, Madrid. España.
}

\begin{abstract}
Resumen
La Pediatría en Atención Primaria en Madrid ha pasado una época complicada. La situación de escasez y aislamiento de los pediatras en los equipos de Atención Primaria nos hace vulnerables para los gestores. La creación en los hospitales de los nuevos contratos mixtos de Facultativo Especialista de Área de Pediatría para cubrir las guardias y las plazas vacantes en los centros de salud ha supuesto una amenaza para la permanencia de los pediatras en el primer nivel asistencial. La contratación de pediatras en Atención Primaria ha permanecido bloqueada por la Administración Sanitaria madrileña durante diez meses para favorecer el desarrollo de estos nuevos contratos. Con la ayuda de sociedades profesionales pediátricas y de ciudadanos, la junta directiva de la Asociación Madrileña de Pediatría de Atención Primaria (AMPap) ha recopilado más de 24000 firmas para el "Manifiesto en defensa del pediatra de cabecera", y tras el debate en la Asamblea de Madrid hemos conseguido desbloquear la contratación de pediatras para los centros de salud.
\end{abstract}

Palabras clave: Pediatras. Atención Primaria. Madrid. Laboral.

\section{Primary Care Pediatrics in Madrid}

Abstract

Primary Care Pediatrics in Madrid has had a complicated time lately. The current situation of isolation and scarcity of pediatricians in primary care teams makes us all vulnerable to managers. The creation of the new "mixed" contracts to cover the current necessities of guards in hospitals as well as vacancies in primary care centers have supposed a threat to the presence of pediatricians at the first level of child care. The hiring of pediatricians in primary care has been

Concha Sánchez Pina: conchasanchezpina@gmail.com

Los autores declaran no presentar conflictos de intereses en relación con la preparación y publicación de este artículo. 
blocked by the Madrid's political administration for 10 months to encourage the growth of these new contracts. The board of the Primary Care Pediatrics Association of Madrid (AMPap) with the help of other pediatrics professional societies and citizens, has collected over 24000 signatures for the Manifesto in defense of the general pediatrician and after the parliamentary debate in the Assembly of Madrid we have achieved to unblock the hiring of pediatricians for the health centers.

Key words: Pediatricians. Primary Care. Madrid. Profession.

\section{Introducción}

La Pediatría de Atención Primaria (PAP) en Madrid ha pasado una época complicada. La situación de escasez, aislamiento e inferioridad de número de los pediatras respecto a los médicos de familia y a las enfermeras en los equipos de Atención Primaria (AP) nos hace vulnerables para los gestores. La creación de los nuevos contratos "mixtos" de Facultativo Especialista de Área de Pediatría para cubrir las guardias en los hospitales y las plazas vacantes en los centros de salud es una amenaza que se cierne sobre los pediatras de Madrid y que podrían generalizarse a todo el país.

\section{Situación}

En octubre de 2008 la Junta Directiva de la Asociación Madrileña de Pediatría de Atención Primaria (AMPap) divulgó los resultados de su encuesta "Situación de la Pediatría en Atención Primaria en Madrid"1 realizada los meses previos a 699 (84\%) pediatras de AP con la colaboración de las juntas directivas de la So- ciedad de Pediatría de Madrid y CastillaLa Mancha y la Sociedad de Pediatría Extrahospitalaria y de Atención Primaria. Las conclusiones de esta encuesta fueron que uno de cada cinco médicos que ocupan una plaza de Pediatría en la Comunidad de Madrid no es pediatra, la mitad $(47,7 \%)$ de los pediatras de Madrid trabajan en turno de tarde con horario fijo de 14:00 a 21:00 horas, y solo el 15\% tenía, en ese momento, turno acabalgado o deslizante. Menos de la mitad de los pediatras encuestados disponían de enfermera con dedicación exclusiva a la Pediatría (44\%). La mayoría de las plazas de Pediatría superaban el número adecuado de tarjetas sanitarias, considerado unas 1000 tarjetas el número adecuado para las sociedades científicas: 22 pediatras $(3,7 \%)$ superaban el cupo máximo de 1500 tarjetas sanitarias asignadas, uno de cada diez pediatras tenía más de 1400 niños y uno de cada tres pediatras tenía más de 1200 niños. Casi el $6 \%$ de las plazas de Pediatría estaban vacantes, y había más de 45000 niños sin pediatra. 
Expusimos los resultados de esta encuesta en nuestra Web, los difundimos en una nota de prensa y los mostramos a la Dirección General de Atención Primaria en varias reuniones. Solicitamos la creación de un grupo de trabajo con profesionales de distintos estamentos para buscar soluciones consensuadas para mejorar el déficit de pediatras y paliar el éxodo de pediatras fuera de nuestra comunidad. Se convocó a las tres sociedades científicas de Pediatría a elaborar un documento coordinado por la Subdirección General de Seguimiento de Objetivos de Atención Primaria, que se concluyó en junio de 2009, llamado "Plan de Mejora ante el Déficit de Pediatras en la Comunidad de Madrid"2. Este plan, iniciado en 2007 por la Consejería de Sanidad madrileña, se ha realizado con la participación de la Sociedad Española de Pediatría Extrahospitalaria y de Atención Primaria, la Sociedad de Pediatría de Madrid y Castilla-La Mancha y la AMPap. Aporta soluciones, acciones priorizadas, plan de actuación, cronograma y evaluación. Se han priorizado las diferentes acciones en función de factibilidad, eficacia y magnitud. Pretende adecuar las revisiones necesarias del niño sano a las necesidades de salud, potenciar el papel de la Enfermería en la atención al niño, favorecer el autocuidado y la autonomía de de- cisión de la población y mejorar la gestión de la demanda en consultas pediátricas. Para hacer más atractiva la AP para los pediatras y no condenarlos a los turnos de tarde con horarios fijos, se ha intentado favorecer la existencia de turnos solapados y deslizantes. Para potenciar el conocimiento de la AP de los especialistas en formación se intentará poner en marcha Comisiones de Docencia entre AP y especializada, establecer la acreditación de tutores de PAP, permitir la acreditación de centros de salud para las rotaciones de MIR-Pediatría independientes de la acreditación de medicina de familia y hacer cumplir la norma de rotación por AP de los residentes de Pediatría los tres meses obligatorios ${ }^{3}$ y su evaluación. Desde la Consejería nos comunican que ya se están poniendo en marcha algunas partes del documento, como favorecer los turnos solapados y deslizantes, refieren que desde el mes de junio de 2008 hasta enero de 2010 de ha incrementado un $32 \%$ (51 plazas) el número de turnos de pediatras con horario flexible, pero sigue habiendo un 43,4\% (369 plazas) con turno de tarde fijo. Para adecuar las revisiones de salud, la atención de la enfermedad aguda y crónica, y mejorar la gestión de la demanda se han creado grupos de trabajo que están estudiando la forma de llevarlo a la práctica. 
Intentando favorecer la existencia de profesionales con actividad hospitalaria y de AP, priorizando la asistencia de los niños y adolescentes por pediatras, la Viceconsejería de Asistencia Sanitaria promulgó el 8 de junio de 2009 la Resolución por la cual se ha creado el puesto de Facultativo Especialista de Área de Pediatría para la prestación de servicios coordinados en los ámbitos asistenciales de Atención Especializada y Primaria, figura que depende exclusivamente de los hospitales y que realiza su trabajo tanto en hospital (guardias y otras actividades) como en AP. Cada plaza vacante de AP se podría asignar temporalmente a dos pediatras "mixtos" que trabajarían en espejo cubriendo entre ambos simultáneamente una plaza del hospital, normalmente de guardias, y una plaza de AP. Esta fue la única oferta posible de trabajo de la sanidad pública de la Comunidad de Madrid para los pediatras recién formados en el año 2009, cerrando así su posible desarrollo profesional como pediatras de AP y bloqueando la contratación a pediatras en las gerencias de $\mathrm{AP}$, reconvirtiendo plazas de los centros de salud en plazas de hospital y produciendo una pérdida de personal y de presupuesto para el primer nivel asistencial. Este nuevo contrato, ideado como con- trato temporal para intentar paliar el déficit de pediatras en las guardias de los hospitales y en las plazas vacantes de AP simultáneamente, ha generado una gran oposición en las sociedades científicas, incluida la Asociación Española de Pediatría, los sindicatos, los colegios profesionales, los pediatras de los hospitales y de AP y los ciudadanos, ya que vulnera la continuidad asistencial, elemento primordial que buscan los padres cuando acuden a nuestras consultas y que lamentarán perder si se generalizara esta nueva figura.

La junta directiva de la AMPap, consciente de la amenaza que estos nuevos contratos suponían para la permanencia de los pediatras integrados en los Equipos de Atención Primaria, escribió con la colaboración de las juntas directivas de la Sociedad de Pediatría de Madrid y Castilla-La Mancha y la Sociedad de Pediatría Extrahospitalaria y de Atención Primaria, varias cartas a la Consejería de Sanidad, comunicados de prensa $y$, aconsejada por la junta directiva de la AEPap, decidió elaborar un Manifiesto en Defensa del Pediatra de Cabecera ${ }^{4}$, redactado por Ángel Carrasco Sanz, vocal de la AMPap y actual Secretario General de la Asociación Española de Pediatría, junto con Ángel Hernández Merino, Director de la Re- 
vista Pediatría en Atención Primaria, quien como miembro de la junta directiva de la AEPap se encargó de redactar un manifiesto que reflejara la problemática de los pediatras a nivel nacional para recoger firmas en todas las autonomías defendiendo la figura del pediatra de cabecera.

El manifiesto de distribuyó por varias sociedades de Pediatría regionales y nacionales, que recopilaron firmas de ciudadanos en apoyo del trabajo de los pediatras en el primer nivel asistencial. Se recopilaron más de 24000 firmas, 5711 electrónicas y 18517 en papel, que se entregaron el día 19 de noviembre en la Consejería de Sanidad, tras lo cual se publicó un nuevo comunicado de prensa5: "Los ciudadanos apoyan nuestro trabajo como pediatras de cabecera en los centros de salud y quieren tenernos a su lado; nos consideran imprescindibles para la salud de sus hijos y nos han mostrado así su apoyo. Los ciudadanos se niegan a que desaparezca su pediatra de cabecera de su centro de salud y a que los pediatras trabajen solo en los hospitales. Les agradecemos su apoyo".

Pero la contratación a pediatras en las gerencias de AP seguía cerrada. La amenaza del peligro de extinción de los pediatras de cabecera permanecía y decidimos, asesorados y apoyados por la junta directiva de la AEPap, denunciar los nuevos contratos "mixtos" a través de un abogado y hablar con todos los partidos políticos de la Asamblea de Madrid que quisieran escucharnos. Contactamos con el Partido Popular, el Partido Socialista de Madrid (PSM) e Izquierda Unida (IU) pero solo el PSM e IU se reunieron con nosotros y decidieron intervenir. Así, el 18 de febrero de 2010 tuvo lugar la Interpelación de la Diputada por IU de la Asamblea de Madrid Caridad Álvarez ${ }^{6}$ y el 11 de marzo la Moción M-1/2010 RGEP.1459, presentada por el Grupo Parlamentario de Izquierda Unida, subsiguiente a la Interpelación 9/2010 RGEP.885, sobre política general en materia de atención sanitaria pediátrica en la Comunidad de Madrid'. Muy pocos días antes, coincidiendo "casualmente" con la publicación en el Boletín Oficial de la Asamblea de Madrid de la Interpelación I-9/2010 RGEP.885 y la Moción M-1/2010 RGEP sobre política general en materia de atención sanitaria pediátrica en la Comunidad de Madrid, la orden de bloquear la contratación a pediatras en las gerencias de AP se revocó.

\section{Recomendaciones}

La situación de escasez, aislamiento e inferioridad de número de los pediatras 
en los centros de salud nos hace prescindibles y vulnerables. Nuestras armas son asociarnos, sindicarnos, buscar alianzas con el resto de sociedades científicas y acudir a manifestarnos en todos los foros disponibles para que nuestra opinión sea tenida en cuenta. Nuestros principales aliados y defensores son los padres de los niños a los que atendemos en nuestras consultas en los centros de salud. Desde que funcionan las listas de comunicación electrónica en nuestras sociedades científicas locales y la lista nacional Pediap, la comunicación entre los pediatras a nivel autonómico y nacional ha mejorado mucho. Debemos formarnos en gestión sanitaria y asumir responsabilidades en los equipos de $\mathrm{AP}$, en las gerencias, en los sindicatos, en los colegios profesionales y en los partidos políticos. Debemos defender nuestro modelo de asistencia pediátrica para asegurar nuestra presencia en los centros de salud integrados en los equipos de AP.

\section{Agradecimientos}

Quiero agradecer de corazón su ayuda a Paloma Hernando, Julia Hernández, Marien Lorente, M. ${ }^{a}$ Jesús Geijo, Ángel Carrasco, Juan Rodríguez y Begoña Rodríguez-Moldes, miembros de la actual junta directiva de la AMPap, y a los miembros de las juntas directivas previas de la AMPap; a Manolo Merino y Juan Bravo, editores de nuestra Web. A Inés Hidalgo y María García-Onieva, compañeras representantes de las otras sociedades científicas e infatigables asistentes a todas las reuniones, quiero agradeceros vuestro incondicional apoyo. A Javier Blanco, Ángel Hernández Merino, Elisa de Frutos, Francisco Javier Soriano Faura, Begoña Domínguez y Juan Ruiz Canela, miembros y presidente de la junta directiva de la AEPap entonces, que nos guiaron en nuestra defensa del pediatra de cabecera, pues sin su estrecha y desinteresada colaboración nada de esto habría sido posible.

\section{Bibliografía}

1. Junta AMPap: Encuesta sobre la situación de la pediatría de atención primaria en Madrid. [Consultado el 29/05/2010.] Disponible en www.am pap.es/profesion/pdf/pediatria_ma drid_2008.pdf.
2. Subdirección General de Seguimiento de Objetivos de Atención Primaria: Plan de mejora ante el déficit de pediatras en la Comunidad de Madrid. [Consultado el 29/05/2010.] Disponible en www.ampap.es/profesion/pdf/2009_05_doc _DG_revisado.pdf. 
3. Orden SCO/3148/2006, de 20 de septiembre, por la que se aprueba y publica el programa formativo de la especialidad de Pediatría y sus áreas específicas. Boletín Oficial del Estado 246 (14-10-2006). [Consultado el 10-07-09.] Disponible en www.boe.es/boe/dias/2006/10/14/ pdfs/A35657-35661.pdf.

4. Hernández Merino A, Carrasco Sanz A. Manifiesto en defensa del pediatra de cabecera. [Consultado el 29/05/10.] Disponible en http://www. ampap.es/profesion/pdf/MANI FIESTO_en_de fensa_del_pediatra_de_cabece ra.pdf.

5. Comunicado de prensa conjunto de sociedades científicas pediátricas: los ciudadanos apoyan mayoritariamente la figura del pediatra de cabecera. [Consultado el 29/05/2010.] Disponible en www.ampap.es/profesion/pdf/20091109 _firmas_manifiesto_np.pdf.
6. Interpelación I-9/2010 RGEP.885 de la Diputada Sra. García Álvarez, del GPIU, al Gobierno, sobre política general en materia de atención sanitaria pediátrica en la Comunidad de Madrid. Boletín Oficial de la Asamblea de Madrid, número 177, 18 de Febrero de 2010. [Consultado el 10/06/ 2010.] Disponible en www.asambleamadrid.es/ Resources/Ficheros/C1/BOAM/BOAM/BOAM_ 8_00177.PDF.

7. Moción M-1/2010 RGEP.1459 presentada por el Grupo Parlamentario de Izquierda Unida, subsiguiente a la Interpelación 9/2010 RGEP. 885, sobre política general en materia de atención sanitaria pediátrica en la Comunidad de Madrid. Boletín Oficial d e la Asamblea de Madrid número 181 del 11 de marzo de 2010. [Consultado el 10/06/ 2010.] Disponible en www.asambleamadrid.es/ Resources/Ficheros/C1/BOAM/BOAM/BOAM _8_00181.pdf. 